\title{
Correction to: Fire Recognition Based On Multi-Channel Convolutional Neural Nefwork
}

Wentao Mao* (D), Wenpeng Wang, Zhi Dou and Yuan Li, Henan Normal University, Xinxiang, China

\section{Correction to: Fire Technology https://doi.org/10.1007/s10694-017- 0695-6}

The original version of this article unfortunately contained a mistake in "Acknowledgement" section. The funding information of "National Natural Science Foundation of China (No. U1704158)" is missing in the original publication. The corrected Acknowledgements section is given below:

\section{Acknowledgements}

This work was supported by China Postdoctoral Science Foundation Special Support (No. 2016T90944), the funding scheme of University Science \& Technology Innovation in Henan Province (15HASTIT022), the funding scheme of University Young Core Instructor in Henan Province (2014GGJS-046), the foundation of Henan Normal University for Excellent Young Teachers (No. 14YQ007) and National Natural Science Foundation of China (No. U1704158).

The original article can be found online at https://doi.org/10.1007/s10694-017-0695-6.

* Correspondence should be addressed to: Wentao Mao, E-mail: maowt.mail@gmail.com 